\title{
PAEDIATRIC ECHO CLINIC SERVICE BY A PAEDIATRICIAN: IS THIS THE WAY FORWARD?
}

\author{
S. Thayath ${ }^{1}$, D. Nguyen ${ }^{2}$ \\ ${ }^{1}$ Paediatrics, Child Development Centre, Addenbrookes Hospital, Cambridge, ${ }^{2}$ Paediatrics, West Suffolk \\ Hospital, Bury St Edmunds, UK
}

Aim: To find out if echo clinic provision can be safely provided by a PEC ( paediatrcian with expertise in cardiology ) in a DGH as an effective screening to tertiary paediatrci cardiology service.

Methods: Retrospective analysis of 181 echoes performed in a 6 month period performed by a PEC. Of the abnormal echocardiograms further analysis were done to see how many had a major abnormality. Further sub analysis was done into this group to see what actions were taken and how many of those were referred to tertiary cardiologist. Referred cases were studied further to see if the diagnosis changed significantly for this group after evaluation by a tertiary cardiologist.

Results: 181 Echoes were done in 6 months by a PEC and his registrar under supervision by the PEC. 77\% of the cases were discharged by the PEC, for a further $16 \%$ plan was made to review the child in a certain period of time and the remaining $7 \%$ were referred onto tertiary cardiologist for further evaluation. Of the 7 $\%$ of cases referred echo finding were not significantly different apart from one case where there was a difference in $\mathrm{M}$ mode measurements but did not make any difference in outcome or management.

Discussion: The results high light that in the hands of a paediatrcian with expertise in cardiology echo clinic service provision can be safely delivered in a district hospital setting and could be an effective measure for reducing unnecessary referrals to tertiary service. 